\title{
Non Hormonal Management of Menstrual Cylce Irregularities
}

\author{
Arif A Faruqui* \\ Department of Pharmacology, Clinical Pharmacologist, A 504, Rizvi Mahal, India
}

Submission: August 16, 2018; Published: September 07, 2018

"Corresponding author: Arif A Faruqui, Department of Pharmacology, A 504, Rizvi Mahal Opp. K.B. Bhabha Hospital, Waterfield road Bandra, India, Email: drfaruqui@gmail.com

\begin{abstract}
Each month the endometrium becomes inflamed, and the luminal portion is shed during menstruation. Aberrations in menstrual physiology can lead to common gynecological conditions, such as heavy or prolonged bleeding. Menstrual dysfunction is defined in terms of bleeding patterns, for example, amenorrhea, menorrhagia or polymenorrhea; ovarian dysfunction for example, anovulation and luteal deficiency; painful menstruation and premenstrual syndrome. Certain characteristics of menstruation can be a reflection of an underlying pathologic process or may predispose a woman to the development of chronic disease. For example, metrorrhagia predisposes to anemia, and the irregular menstrual cycles associated with PCOS (see PCOS) can predispose a woman to infertility, diabetes and consequently, heart disease.

What is it, in this age of life-saving antibiotics, hormonal therapy, surgeries and other seemingly miraculous medical therapies that causes so many individuals to seek therapies outside of conventional medicine? Conventional medicine may be at its best when treating acute crises, but for the treatment of chronic problems it may fall short of offering either cure or healing, leading patients to seek out systems of treatment that they perceive as addressing the causes of their problem, not just the symptoms. According to WHO, experience has shown that there are real benefits in the long term use of whole medicinal plants and their extracts, since the constituents in them work in conjunction with each other. Globally, it is estimated that $70 \%$ of all health care is provided by traditional, nonconventional medicine. Vitex agnus castus or chaste tree is one such herb which has a long history of use for common gynecological complaints.
\end{abstract}

Keywords: Vitex agnus castus; Menstrual cycle irregularities; PMS; Hyperprolactinemia; Non Hormonal

Abbreviations: GnRH: Gonadotropin Releasing Hormone; FSH: Follicle Stimulating Hormone; LH: Luteinizing Hormone; TRH: Thryotrophin Releasing Hormone; VAS: Visual Analog Scale

\section{Introduction}

The menstrual cycle is one of the most exquisite displays of biological rhythm. It is a fascinating combination of positive and negative feedback controls involving the hypothalamus, pituitary, thyroid, adrenals, ovaries and uterus [1].

Hormones and Their Roles in Regulating Menstrual Cycle

The actual centre of control is the hypothalamus, which produces a gonadotropin releasing hormone $(\mathrm{GnRH})$ that stimulates the anterior pituitary to release the gonadotropins follicle stimulating hormone (FSH) and luteinizing hormone (LH). Pulsatile secretion of GnRH is necessary for the pituitary to respond with adequate production of LH and FSH [2].

FSH is the primary hormone responsible for the maturation of follicles into fertile ova and the increased production of estrogen by the ovaries. LH causes release of the ovum, conversion of the follicle into the corpus luteum, and the subsequent production of progesterone [2].

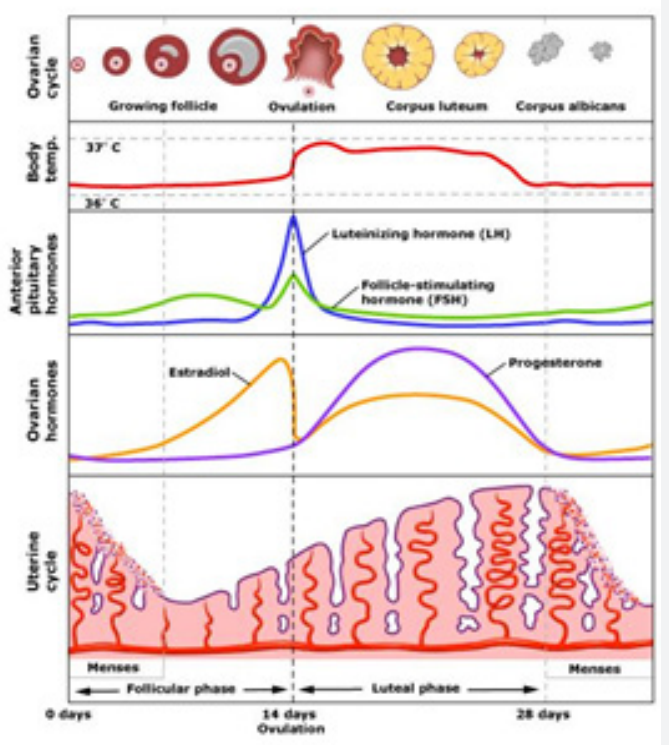

Figure 1: Menstrual cycle- Summary. 
At mid-cycle, estrogen is at its peak and progesterone begins to rise. It is at this point that FSH levels decrease and LH levels surge to cause ovulation. In the ovary, the corpus luteum produces progesterone. This hormone ensures sufficient blood supply to the endometrium so that the fertilized ovum can establish itself in the uterus. If fertilization does not occur, the corpus luteum recedes, hormone production decreases, the endometrium is not sufficiently supplied with blood and menses occurs. FSH and LH levels decline until menses and the beginning of a new menstrual cycle (Figure 1) [2].

\section{Incidence of Gynecological Disorders}

Excessive or inappropriately timed bleeding from the vagina is one of the most common complaints for which women seek advice from medical healthcare providers [3]. 92\% of women in India suffer from gynecological problems. In a study conducted among the women of rural India, $60.6 \%$ were having menstrual diseases as one of the common gynecological diseases [4].

One of the major issue encountered during surveys have been underreporting. Bang et al found that only 7-8\% of the women had ever had a gynecological examination in the past, even though 55\% were aware of having gynecological disorders [4]. Normal menstruation requires integration of the hypothalamicpituitary-ovarian axis with a functional uterus, a patent lower genital outflow tract and a normal genetic karyotype of 46XX [3].

\section{Prevalence of Common Menstrual Disorders (Indian Data)}

Cyclic symptoms in women of reproductive age have been recognized for thousands of years. Premenstrual syndrome (PMS) is one of the most common disorders of women of reproductive age. The incidence of PMS peaks among women age 30 to 40, but studies have shown that adolescents frequently suffer the effects of PMS as well [5].

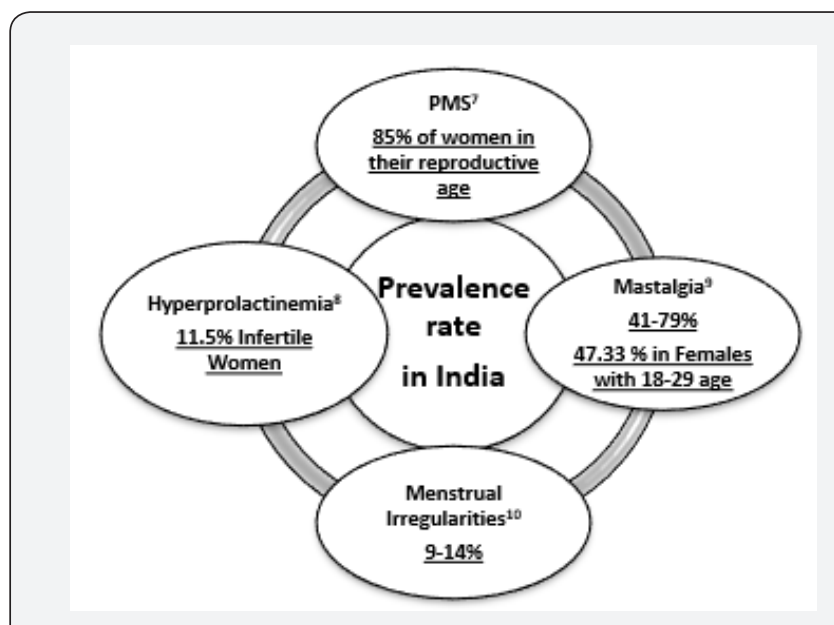

Figure 2: Prevalence of menstrual cycle irregularities, PMS, Hyperprolactinaemia and Mastalgia in Indian females.

Hyperprolactinemia is a common endocrine disorder of hypothalamic-pituitary ovarian axis affecting the reproductive functions [6]. Prevalence of mastalgia is highly variable and reported with a rate of $51-54 \%$ in adult Indian urban population [7]. Prevalence rate of various menstrual abnormalities is depicted below in Figure 2.

\section{Menstrual cycle irregularities and its causes}

Various underlying disease conditions like hypothyroidism, pituitary adenoma, PCOS, hyperprolactinemia, drug induced (dopamine antagonists like antipsychotics, antidepressants etc.) can lead to menstrual cycle irregularities. Hormonal imbalance due to various underlying causes is one of the major etiologies behind menstrual cycle irregularities (Figure 3).

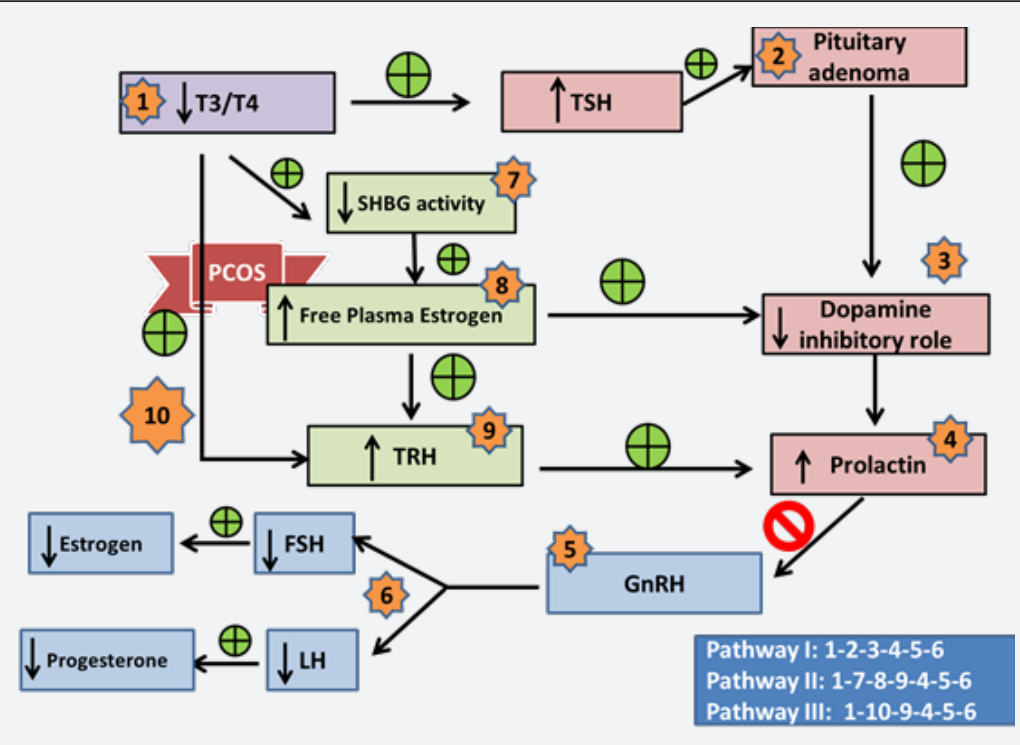

Figure 3: Various hormonal pathways leading to menstrual cycle irregularities.

Pre-Menstrual Syndrome (PMS)

PMS is a psycho-neuroendocrine disorder of unknown etiology, often noticed just prior to menstruation. There is cyclic appearance of large number of symptoms during the last 7-10 days of the menstrual cycle. 


\section{Pathophysiology}

The exact cause is not known but following hypothesis is postulated:

a) Alteration in the level of estrogen and progesterone starting from the midluteal phase. Either there is altered estrogen: progesterone ratio or diminished progesterone level.

b) Neuroendocrine factors

c) Serotonin is an important neurotransmitter in the CNS. During the luteal phase, decreased synthesis of serotonin is observed in women suffering from PMS.

d) Endorphins: The symptom complex of PMS is thought to be due to the withdrawal of endorphins (neurotransmitters) from CNS.

e) GABA suppresses the anxiety level in the brain. Medications that are GABA agonist are effective.

f) Psychological and psychosocial factors may be involved to produce behavioral changes.

g) Others: Variety of factors has been mentioned to explain the symptom complex of PMS. These are thryotrophin releasing hormone (TRH) prolactin, renin, aldosterone, prostaglandins and others.

\section{Clinical Features}

PMS is more common in women aged 30-45. It may be related to childbirth or a disturbing life event. The symptoms of PMS are depicted below in Figure 4.

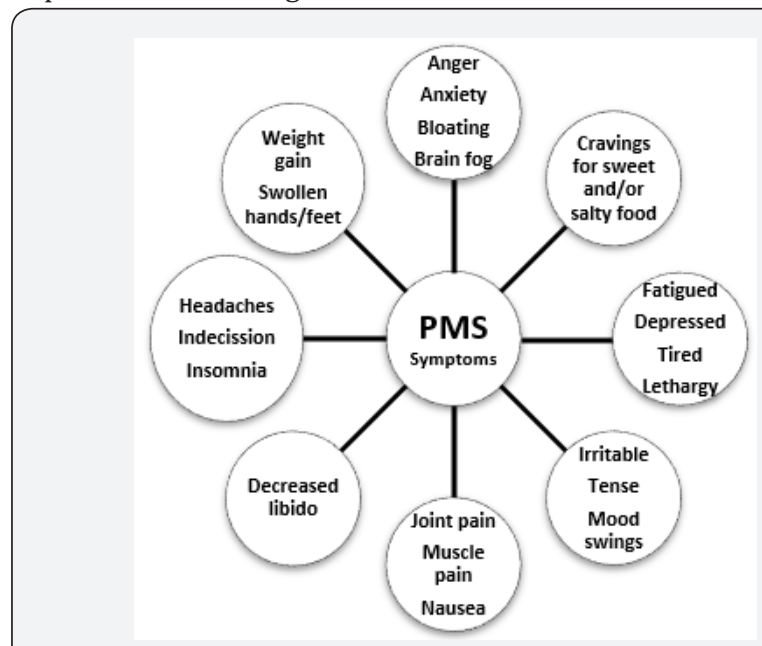

Figure 4: Symptoms of Pre-menstrual syndrome.

\section{Hyperprolactinaemia}

Prolactin is a peptide hormone produced by the anterior pituitary gland that is primarily associated with lactation and plays a vital role in breast development during pregnancy [8]. Prolactin inhibits GnRH (gonadotropin releasing hormone) pulse secretion. Gonadotropin levels are suppressed. Hyperprolactinaemia inhibits ovarian steriodogenesis.
Hyperprolactinaemia causes secondary amenorrhea in about $30 \%$ of women. There is anovulation and hypogonadotropic hypagonadism [8].

\section{Signs and symptoms}

Hypoestrogenism with anovulatory infertility and a decrease in menstruation, amenorrhea, menstrual irregularities, women who are not pregnant or nursing may begin producing breast milk, loss of libido, breast pain, vaginal dryness.

\section{Current Therapy for Management of Menstrual Cycle Irregularities and PMS Includes}

(a) Hormonal contraceptives: These are the drugs that prevent ovulation and may be effective in relieving physical symptoms.

- $\quad$ Combined oral contraceptives (COC) are preferred in females who do not desire to conceive whereas

- $\quad$ Single progesterone pills are preferred in those with a desire of pregnancy

(b) Antidepressants- These drugs can help lessen mood symptoms.

(c) Anti-anxiety drugs- If anxiety is a major PMS symptom, these drugs may be used.

(d) Non-steroidal anti-inflammatory drugs (NSAIDs) can help reduce pain. Long term use of NSAIDs is not recommended.

(e) Diuretics are drugs that help the body to lose excess water through the kidneys. These medicines have been used to reduce weight gain, breast swelling, and bloating associated with PMS.

Limitations of Existing Therapy for Menstrual Cycle Irregularities and PMS

a) Hormonal/OCTs/COCs (Progesterone alone or combination with estrogen): Possibility of unwanted 'premenstrual symptoms' including (bloating, oedema, headache, depression and reduced libido), Irregular breakthrough bleeding, development of blood clots, gall bladder disease [9].

b) Androgens (Danazole): Weight gain, deepening of voice \& Monitor lipid profile and liver function [2].

c) Dopamine agonists (Bromocriptine, Cabergoline): 28$55 \%$ have reported of nausea, vomiting, abdominal pain, headache, postural hypotension, dizziness, drowsiness $[10,11]$.

\section{Role of Herbal Medicine in Gynecological Disorders}

Botanical treatment can provide treatment and support for numerous fertility-related problems, such as hormonal dysregulation, thyroid and adrenal disorders, genitourinary infections, immune dysregulation, and stress-related problems with hormone like effects and minimal side effects [12]. 
Vitex agnus castus is one such herb which has a long history of use for gynecologic complaints. Chaste berry should be considered a first-line botanical therapy for infertility associated with secondary amenorrhea, hyperprolactinemia, and luteal insufficiency [12]. It is approved in various European countries since year 1968 for various indications like: menstrual cycle irregularities, PMS, Mastalgia.
Vitex agnus castus, also known as chaste tree, is a shrub with finger-shaped leaves and slender violet flowers. Vitex agnus castus grows in creek beds and on river banks in valleys and lower foothills in the Mediterranean and Central Asia [2].

\section{Vitex agnus castus constituents}

Essential oils, Iridoid glycosides, Flavonoids, Diterpenes, Essential fatty acids.

\section{Role of Vitex Agnus Castus (Chaste Tree) in Regulating Hormonal Imbalance (Figure 5)}

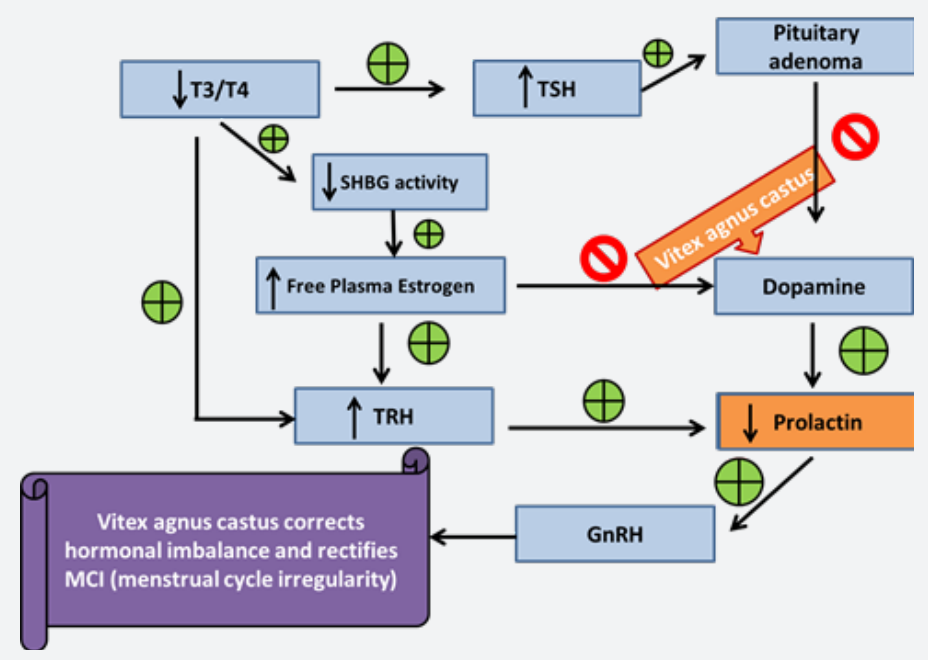

Figure 5: Role of VAC (Vitex agnus castus) in regulating hormonal imbalance.

a) Chaste regulates prolactin levels, enhance corpus luteum development and correct relative progesterone deficiency [12].

b) It modulates the anterior pituitary's production of luteinizing hormone ( $\mathrm{LH})$, while mildly inhibiting follicle stimulating hormone (FSH) [12].

c) It downregulates the production of excess prolactin in hyperprolactinemia via dopaminergic activity [12].

d) Chaste berry reduces thyroxin-releasing hormone (TRH)-induced prolactin release (essentially a pituitarythyroid axis problem) [12].

e) It normalizes shortened luteal phases, corrects luteal phase progesterone deficiencies and reduces PMS symptoms in women with luteal phase defects caused by latent hyperprolactinemia [12].

Clinical Evidences of Use of VAC in Various Gynecological Conditions

In an observational study conducted on 211 females with complain of Polymenorrhea, Oligomenorrhoea, Amenorrhea, Dysmenorrhea, Menometrorrhagia; the use of $20 \mathrm{mg}$ VAC for 3 menstrual cycles has shown improvement in menstrual cycle irregularities among $79-85 \%$ also $60-88 \%$ females showed improvement in menstrual bleeding disorders [13].

Another study conducted on 2,447 women with a variety of menstrual problems, use of VAC has shown improvement in symptoms by both patients and physicians. Also the safety profile of herb was excellent with only $2.3 \%$ reporting minor side effects [14].

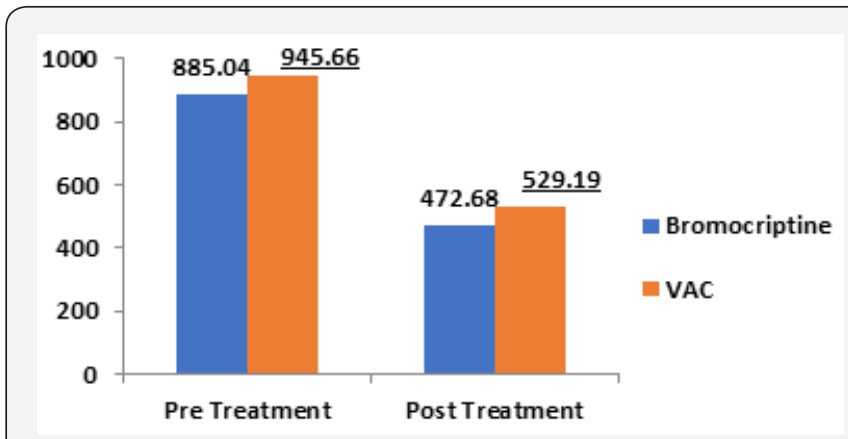

Figure 6: Reduction of serum prolactin levels by VAC and bromocriptine.

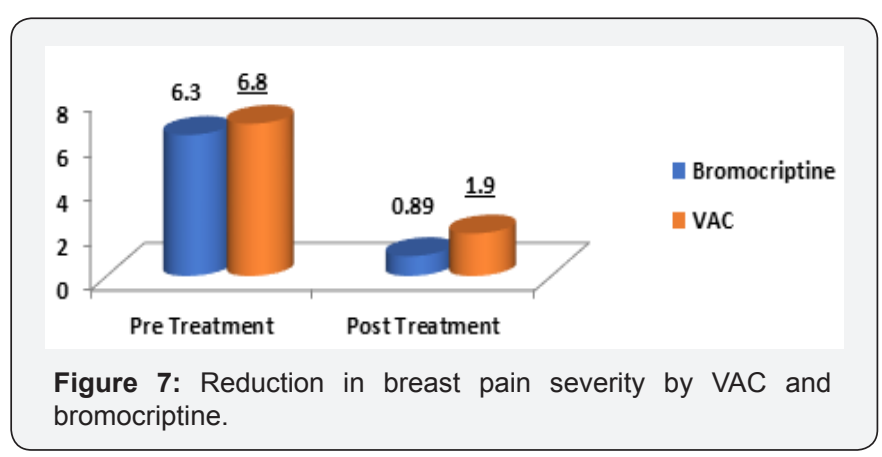


In a randomised active controlled study conducted on 80 females with hyperprolactinemia and cyclic mastalgia, efficacy of both drugs (VAC and bromocriptine) was assessed by measuring serum prolactin levels on day 5-8 of menstrual cycle and pain severity for mastalgia was evaluated using visual analog scale
(VAS) [15]. (Figure 6\&7) Multicentric trial of VAC conducted on 1634 patients with symptoms of PMS demonstrated decrease in number of symptoms by $93 \%$ females in turn qualifying the efficacious and safe use of herb in females with PMS symptoms [16].

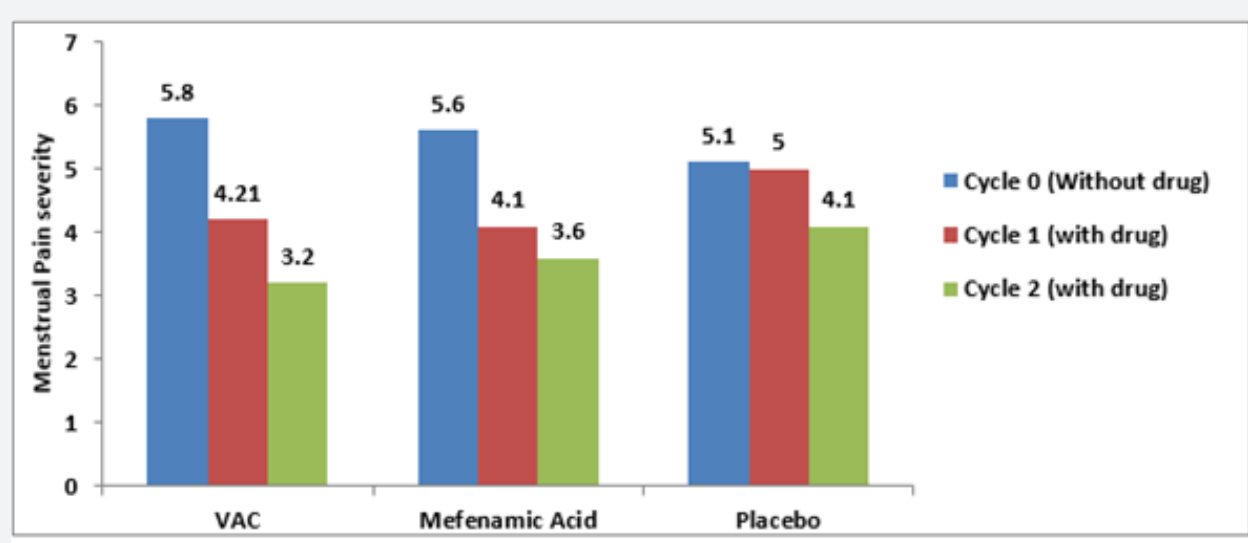

Figure 8: Comparative efficacy of VAC, mefenamic acid and placebo in decreasing menstrual pain severity.

In a double blind study conducted on 80 female students with primary dysmenorrhea, authors had tried to evaluate the efficacy of VAC, mefenamic acid and placebo for duration of 3 menstrual cycles. Severity of dysmenorrhea was assessed using pain severity measurement tool. Menstrual pain severity was lower with VAC as compared to mefenamic acid and placebo [17] (Figure 8).

When compared with analgesics (NSAID), hormones, or SSRi (selective serotonin reuptake inhibitors), which help only in symptom alleviation, VAC is an effective treatment for relieving not only the physical but also the mental symptoms of PMS treatment.

In a randomized controlled trial conducted in 100 infertile cases with PCOS, females were randomised to either clomiphene citrate or combination of VAC and clomiphene citrate to evaluate the pregnancy rate. Pregnancy rate encountered in group receiving clomiphene citrate plus VAC was $60 \%$ while only $30 \%$ was observed clomiphene citrate group which confers that the pregnancy rate can be doubled by adding VAC to conventional therapy while treating infertile females [18].

\section{Discussion}

In spite of the significance of menstruation in women's lives and the high incidence of menstrual related health problems in society, there is surprisingly little epidemiologic evidence on menstrual disorders and associated risk factors. Certain characteristics of menstruation can be a reflection of an underlying pathologic process or may predispose a woman to the development of chronic disease. For example, Metrorrhagia predisposes to anemia, and the irregular menstrual cycles associated with PCOS (see PCOS) can predispose a woman to infertility, diabetes and consequently, heart disease [12].
Each treatment option has overt and hidden costs, including emotional, physical, and financial burdens, often without justification because of lack of successs [12]. Oral progestogens are the most widely used medical treatment for dysfunctional uterine bleeding (DUB)/ menstrual cycle irregularities. Despite this, their efficacy is poorly established, both for ovulatory and anovulatory [12]. Disadvantages of hormonal therapy arises the need of alternative treatment with similar or better efficacy and minimal ill-effects.

Vitex agnus castus being non-hormonal therapy has been used for centuries in menstrual cycle irregularities, hyperprolactinaemia \& premenstrual syndrome and also used in clinical practice to assist with withdrawal from hormone therapy. Efficacy \& Tolerability of VAC is evidenced by $>30$ clinical trials with $>10,000$ patients.

Vitex agnus castus is approved by German Commission E and European Medicine Agency's (EMA) for management of menstrual cycle irregularities, PMS and hyperprolactinemia. Up to $85 \%$ response rate has been documented in menstrual cycle irregularities like polymenorrhea, Oligomenorrhea and amenorrhea while up to $88 \%$ response rate has been observed in menstrual bleeding disorders like dysmenorrhea, intermenstrual bleeding, hypermenorrhea, menometrorrhagia, ovulation/pre/postmenstrual bleeding.

\section{Conclusion}

In view of the associated adverse effects of current therapy, the need of the hour is to explore alternative options which have at par efficacy \& superior safety profile compared with modern medicine drugs. Vitex agnus castus having efficacy similar to conventional drugs with relatively superior safety profile is one such non-hormonal therapy which can be recommended as first line therapy in the management of menstrual cycle irregularities 
\& infertility associated with secondary amenorrhea, hyperprolactinemia, and luteal insufficiency.

\section{References}

1. Guilliams TG (2001) Female cycle difficulties. Non-invasive Diagnosis and Natural Treatment Options. The Standard 4(2).

2. Brown DJ (1994) Vitex agnus castus monograph. Quarterly Review of Natural Medicine pp. 111-121.

3. Howkins, Bourne Shaw's (2004) Disorders of Menstruation. Textbook of gynecology, In: (13 ${ }^{\text {th }}$ edn), pp. 277-290.

4. Bang RA, Bang AT, Baitule M, Choudhary Y, Sarmukaddam S, et al. (1989) High prevalence of gynaecological diseases in rural Indian women. Lancet 1 (8629): 85-88.

5. Joseph LM (1999) A Natural Approach to Management. Applied Nutritional Science Reports 5(6): 1-8.

6. Hariharan C, Agrawal S (2013) Prevalence of Hyperprolactinaemia in infertile cases and its correlation with TSH in a rural set up hospital. Int J Reprod Contracept Obstet Gynecol 2(4): 626-630.

7. Raghunath S, Raghuram N, Ravi S, Ram NC, Ram A, et al. (2015) Prevalence of mastalgia in young Indian females. J Health Res Rev 2(3):108-111.

8. (2013) DC Dutta's Text book of gynecology. In: ( $\left.6^{\text {th }} e d n\right)$, Amenohrrea, pp. 464-465.

9. Hickey M, Lester S (2003) The management of menstrual disorders in early reproductive life. The Obstetrician \& Gynaecologist 5: 136-141.
10. Johnston DG, Prescott RW, Kendall-Taylor P, Hall K, Crombie AL, et al. (1983) Hyperprolactinemia. Long-term effects of bromocriptine. Am J Med 75(5): 868-874.

11. Motazedian S, Babakhani L, Fereshtehnejad SM, Mojthahedi K (2010) A comparison of bromocriptine \& cabergoline on fertility outcome of hyperprolactinemic infertile women undergoing intrauterine insemination. Indian J Med Res 131: 670-674.

12. Aviva R (2010) Botanical medicine for women's health. Chapter 12 (Fertility challanges), pp. 334-346.

13. Eltbogen R, Litschgi M, Gasser U, Nebel S, Zahner C, et al. (2014) Vitex Agnus-Castus extract (Ze 440) improves symptoms in women with menstrual cycle irregularities. Planta Med, pp. 80-SL19.

14. Loch $E$ (1991) The treatment of menstrual disorders with Vitex agnuscastus tincture. Der Frauenarzt 32: 867-870.

15. Kilicdag EB, Tarim E, Bagis T, Erkanli S, Aslan E, et al. (2004) Fructus agni casti and bromocriptine for treatment of Hyperprolactinaemia and mastalgia. Int J Gynaecol Obstet 85(3): 292-293.

16. Loch EG, Selle H, Boblitz N (2000) Treatment of premenstrual syndrome with a phytopharmaceutical formulation containing Vitex agnus castus. J Womens Health Gend Based Med 9(3): 315-320.

17. Shobeiri F, Zeraati F, Mansouri Z, Araghchian M, Nazari M, et al. (2012) The Comparative Effect of Herbal Extract of Vitagnus and Mefenamic Acid on Primary Dysmenorrhea. ZJRMS 14(5): 30-33.

18. Elsayed (2013) Agnucaston and Clomiphen Citrate in Infertile Patients with Polycystic Ovaries. J IVF Reprod Med Genet 1: 2

\begin{tabular}{|l|}
\hline \multicolumn{1}{|c|}{ Your next submission with Juniper Publishers } \\
will reach you the below assets \\
- Quality Editorial service \\
- Swift Peer Review \\
- Reprints availability \\
- E-prints Service \\
- Manuscript Podcast for convenient understanding \\
- Global attainment for your research \\
- Manuscript accessibility in different formats \\
( Pdf, E-pub, Full Text, Audio) \\
- Unceasing customer service \\
Track the below URL for one-step submission \\
https://juniperpublishers.com/online-submission.php \\
\hline
\end{tabular}

